\title{
SSIMS Molecular Selective Imaging: A New Diagnostic Tool To Investigate Metal Passivators In Scrapped Transformers
}

\author{
M. Facciotti*, P.S. Amaro, R.C.D. Brown, P.L. Lewin, \\ J.A. Pilgrim \\ University of Southampton \\ Southampton, Hampshire \\ SO17 1BJ, UK \\ *facciotti.marco@gmail.com
}

\author{
G. Wilson and P.N. Jarman \\ National Grid \\ National Grid House, \\ Warwick Technology Park, \\ Gallows Hills, Warwick CV34 6DA, UK
}

\begin{abstract}
In every high voltage grid, the replacement of assets is a slow but steady process that guarantees long-term reliability of the power distribution networks. The prioritisation of such interventions is based on complex rating criteria specifically designed to highlight potential issues of certain machines (or families of machines) in need of care. Given the tremendous costs of operations such as the replacement of a power transformer it is not surprising to observe constant efforts devoted to finding new and better monitoring and diagnostic tools. These are capable of delivering invaluable information about the conditions of a transformer, in service or after it is either failed or proactively scrapped, allowing a better comprehension of underlying chemical-physical phenomena occurring. Ideally, advanced monitoring and diagnostic tools should ultimately result in improved rating parameters to be applied in the evaluation of future interventions. This feasibility study evaluates the use of static secondary ion mass spectrometry (SSIMS) molecular selective imaging as a diagnostic tool for power transformer. SSIMS is herein demonstrated to be able to assess, without doubts, the presence and integrity of the passivation layer produced by $\operatorname{Irgamet}^{\circledR} 39$ on copper surfaces in laboratory tests. The technique has also been tested on real samples collected from one of the phases of a $400 / 275 \mathrm{kV}$ autotransformer proactively scrapped and proved to be applicable without significant sample preparation. The possibility of its use in the study of the distribution of Irgamet ${ }^{\circledR} 39$ across the windings is also demonstrated. Further development of SSIMS as a diagnostic tool would be significantly increasing the grade of detail at which scrapped/failed units could be inspected. A better understanding on how the operating conditions or the design of a transformer can affect the anticorrosion protection layer at the molecular level would open the way to significant ad hoc improvements of both operative guidelines and rating criteria for power transformers
\end{abstract}

Keywords-transformer; scrapping; diagnostics; passivator; corrosive sulphur; SSIMS.

\section{INTRODUCTION}

Failures directly or indirectly linked to 'corrosive sulphur' in oil-filled electrical power transformers have been object of interest for the scientific community many years now. Both chemists and engineers studied this phenomenon trying to develop new mitigation strategies and diagnostic tools to minimize the risks and prevent the catastrophic consequences of failures [1]-[4]. Regarding chemistry-related efforts, many well-known analytical techniques have been successfully applied mainly on the more easily accessible oil samples, such as chromatography [1], [5], [6], spectroscopy [7]-[10] and dissolved gas analysis [4], [11], [12]. Fewer techniques have been applied to the study of copper and paper [13], [14]. This is ultimately caused by the obvious sampling difficulties one would experience trying to get these materials out of a transformer in service (even if not live). The scrapyard is often the fulcrum of the diagnostic process on windings and solid insulation although, unless in the case of proactively scrapped units, it is generally after the failure occurred. Even at this point, however, visual assessment of the materials is usually the first level of investigation only eventually followed by laboratory tests like DP of paper or electron microscopy [1], [8], [15], [16]. The aim of this paper is to show the unique imaging capabilities of an advanced surface chemistry analytical technique, static secondary ion mass spectrometry (SSIMS), in the study and diagnostics of corrosion and passivation phenomena occurring at the copper surface of transformer windings, in order to increase the fundamental understanding of the chemical process involved and pave the way for further improvement in the management of such a complex and dangerous issue.

\section{EXPERIMENTAL PROCEDURES}

Samples from the winding of the transformer investigated were collected during the scrapping by qualified engineers. Sample preparation for the chemical analysis of the surface of the metal simply consisted of three steps. Firstly, samples were cut to size (ca. $1 \mathrm{~cm}^{2}$ ) using a bull cutter to minimize bending and dust formation. All layers of insulating paper were removed and copper samples were thoroughly washed three times with fresh cyclohexane to remove all oil traces. The production of the sample to study the consequence of surface breakdown on the passivation layer was more complex. A portion of polyurethane-insulated copper microwire $(99.99 \%, \varnothing 25 \mu \mathrm{m}, 5 \mu \mathrm{m}$ insulation thickness, Goodfellow Cambridge Ltd.) was sealed into glass. Fig. 1a 
shows a glass pipette containing the wire, connected to a vacuum pump, being melted by a glowing resistor wire. This produced a bubble-free glass embedded copper wire that was then polished, using sand paper and abrasive paste, down to 1 $\mu \mathrm{m}$ and sonicated in deionized water for 5 minutes to obtain a flat mirror-finished copper surface. Two of these samples were prepared. The pipettes were then cut down to a total length of $15 \mathrm{~mm}$ and placed in $20 \mathrm{~mL}$ PTFE crimp-sealed headspace glass vials together with $15 \mathrm{~g}$ of unused Gemini X insulating transformer oil, free from detectable DBDS, spiked with 100 ppm of passivator Irgamet ${ }^{\circledR} 39$ (BASF). Vials were then placed in fan oven for 24 hours at $70{ }^{\circ} \mathrm{C}$ to allow passivation to occur. Afterwards, samples were thoroughly washed three times with fresh cyclohexane to remove all oil traces. One sample was kept as reference while the other underwent surface breakdown. Fig. $1 \mathrm{~b}$ shows the setup used to generate the surface breakdown on the sample. It can be noted how the copper wire was earthed and kept standing by means of some Sn-alloy soldering wire. As the experiment was to be performed in oil, to better simulate real operating conditions, and with optimal alignment, once the needle was positioned over the sample, a drop of Gemini X oil was deposited between the two allowing surface tension only to keep it in place, as shown in Fig. 1c. The voltage breakdown was approximately $19 \mathrm{kV} \pm 2 \mathrm{kV}$, with the voltage ramp rate set to $50 \mathrm{~V} / \mathrm{s}$ and an electrode distance set to $1 \mathrm{~mm}$. The test was repeated a few times to increase the chances of identifying the breakdown site in the copper wire cross-section surface using SSIMS. Although the setup appears to be a needle-to-plane arrangement, due to the size of the cross-section of the copper wire in reality the field arrangement is more like a needle-toneedle setup. No further treatment was required on the sample rather than the already described degreasing treatment with cyclohexane.

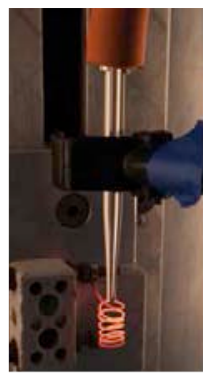

(a)

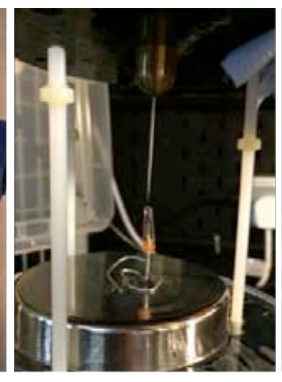

(b)

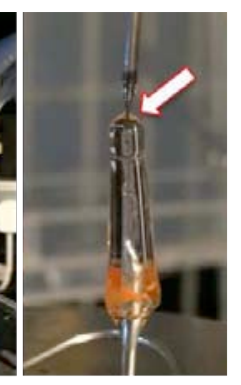

(c)
Fig. 1. Graphical summary of the surface breakdown experiment showing glass pipette containing copper wire, under suction, melting using a glowinf resistor wire (a); overview of the setup used (b); detail of the alignment between sample and needle, connected by a drop of Gemini X oil (arrow in c).

\section{Static secondary ion mass spectrometry imaging}

Samples described in this work were analysed by SSIMS (static secondary ion mass spectrometry) using an Ion-ToF 'ToFSIMS IV' instrument. SIMS is a particular kind of mass spectrometry that allows the study of a material on the molecular level using a focused primary ion or to sputter atoms and molecules away from the surface of a sample, in the form of charged species, as a consequence of elastic and inelastic scattering interactions. These are then analysed by a mass spectrometer. The operational mode used in the applications described in this paper is named static. It uses a low energy primary beam for a more delicate sputtering of the surface of the sample minimizing charging effects and fragmentation of organic molecules, which can be more easily observed. The samples for analysis were handled using clean stainless steel tweezers at all times. The $\mathrm{Bi}_{3}{ }^{2+}$ ion source was used in all cases reported. The main chamber pressure during analysis was less than $\approx 5 \times 10^{-7}$ mbar from a base pressure of $\approx 5 \times 10^{-9}$ mbar. Negative polarity spectra were collected using a $500 \mu \mathrm{m}^{2}$ analysis area in each case. The total ion dose for each profile was $\approx 2 \times 10^{12}$ ions $\mathrm{cm}^{-2}$, which was within the accepted ion dose limit for static SIMS of $5 \times 10^{12}$ ions $\mathrm{cm}^{-2}$. The spatial resolution achieved in the images was $\approx 4$ $\mu \mathrm{m}$ and the mass resolution $(\mathrm{m} / \Delta \mathrm{m})$, was around 5000 . Each of the composed images is generated by superposition of the three ion channels collected in red-green-blue mode (RGB) representing copper, passivator and sulphur respectively. Each one of the separate feeds shows the intensity, scaled from 0 to 225 for display purposes, of the relative species. When a specific pixel is shared between multiple feeds the resulting colour is the addition of the three components, scaled by their respective intensities. So, if all three detected species were to show maximum intensity (i.e. 255) these would combine to give a white pixel. Therefore, whichever species shows the most intensity will tend to dominate the final colour of any given pixel.

\section{RESULTS AND DISCUSSION}

\section{A. Reaction for the formation of the passivation layer on copper conductors}

The addition of a passivator such as Irgamet $^{\circledR 3} 3$ in transformer oil sets into motion a series of chemical transformations that ultimately result in the formation of a passivation layer that is capable of withstanding the attack of reactive sulphur species (e.g. DBDS, dibenzyl disulphide). Irgamet ${ }^{\circledR} 39$ comprises two different moieties. The first one is heteroaromatic, a tolyltriazole, which is the only surface active part of the molecule acting as a metal passivator; the second is an aliphatic amine that only serves as a solubilisation aid for the former one, otherwise insoluble in insulating oil. In presence of copper, Irgamet ${ }^{\circledR 39}$ undergoes a retro-Mannich reaction that allows the liberation the active tolyltriazole, thus free to bind to the metal surface producing the passivation layer, as shown in Fig. 2.

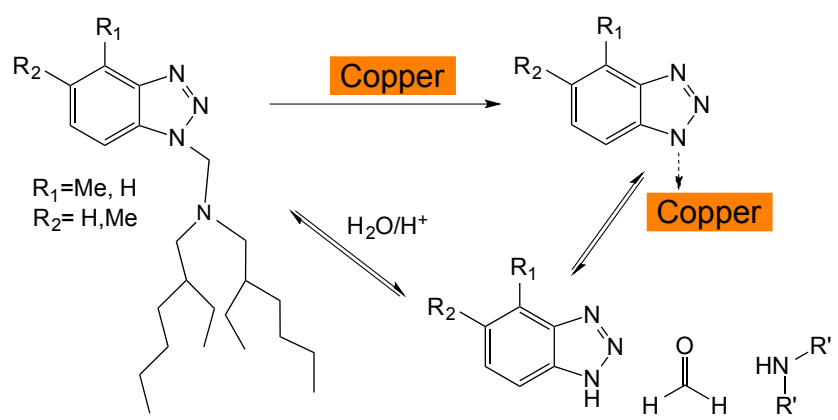

Fig. 2. Chemical structure of Irgamet $^{\circledR 39}$ (top left) and retro-Mannich reaction to form the passivation layer on the surface of copper. 
The concentration of passivator Irgamet ${ }^{\circledR} 39$ normally recommended in transformer applications is $100 \mathrm{mg} / \mathrm{kg}$. Confirmation of the hypothesized mechanism was found thanks to SSIMS in the detection, on the passivated metal surfaces analyzed, of the molecular fingerprint of tolyltriazole. Signal from the corresponding molecular ion in negative polarity mode, a deprotonated tolyltriazole in the form [M-H] and $\mathrm{m} / \mathrm{z}=-132$, was observed on all passivated samples.

\section{B. Distribution of passivator in a 400/275 kV autotransformer}

SSIMS imaging was tested as new diagnostic tool for postmortem inspection of power transformer windings. As an example, samples collected from one phase of a proactively scrapped 400/275 kV autotransformer were analysed. The transformer was known to have suffered prolonged overheating of the top discs, possibly due to design issues (OFAF/ODAN), and to have been manipulated in the thermostat regulations of its cooling fans, due to noise complaints. Results of the measurements are shown in Fig. 3.

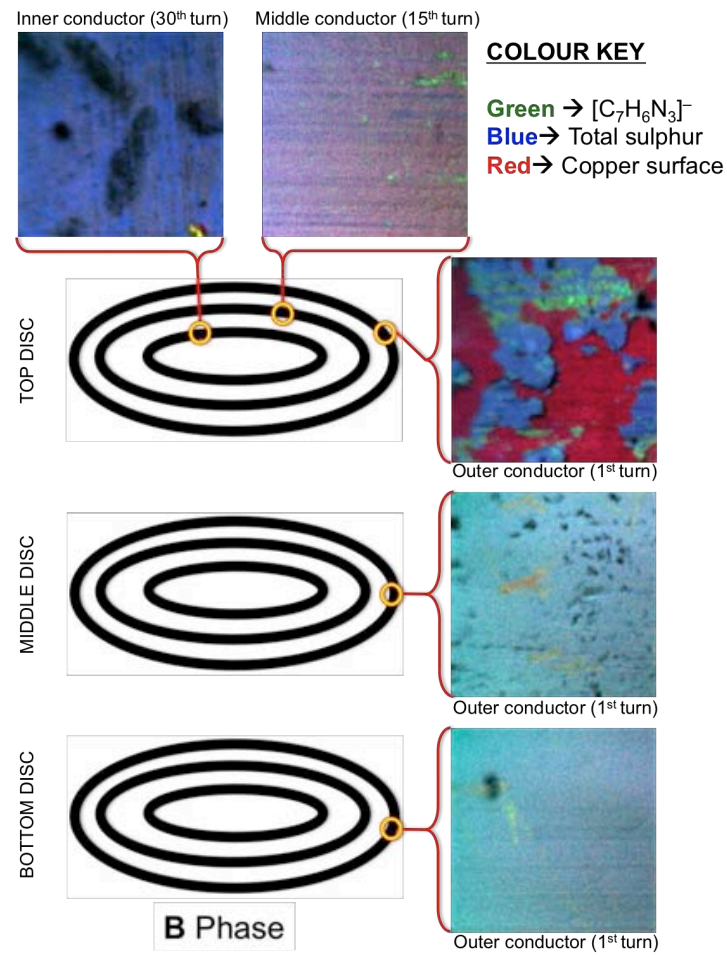

Fig. 3. Schematic representation of the location of the copper samples analyzed by SSIMS in the bottom, middle and top winding discs. Molecular selective imaging of each sample is shown as final composed image.

A total of five bare copper samples were analyzed. The main difference between them was their relative position in the transformer winding and therefore the temperatures to which they were subjected during the operational life of the transformer. The appearance of the metal surfaces from the colder bottom and middle discs were quite similar, a rather uniform false turquoise, due to mixed contribution of both green and blue pixels to the final composed image, representing tolyltriazole and sulphur-containing ions respectively. Sulphur was obviously present since passivators are only added to corrosive oils, potentially capable of damaging copper parts. The three samples from the top discs were very different from the others and between each other, suggesting a more complex influence of temperature. No substantial presence of passivator was detected, suggesting its distruction due to prolonged thermal stresses. The inner regions of the top disc appeared heavily corroded as mostly signal from sulphur corrosion by-products is collected, while no evidence of underneath copper was detected suggesting a well-attached corroded layer. In the middle region more exposed copper was visible, together with traces of passivator. The outer region of the top disc, however, seemed to be the most affected by advanced corrosion signs: exposed copper (potentially vulnerable to corrosion) was predominant together with poorly attached and flaky sulphur-rich formations constituted of corrosion by-products (e.g. copper sulphide). There could be no doubt in considering this region of the winding as the most probable source of the copper sulphide contamination of the transformer, as a consequence of the thermal stresses at which the passivation layer was subjected during the operational life of the transformer. This also confirmed the vulnerability of passivation in case of prolonged exposure to abnormal high temperatures.

\section{Study on the effect of a surface breakdown on the passivation layer}

SSIMS imaging was also applied to establish whether the development of a surface breakdown on the windings was able to cause any damage to the passivation layer, hence increasing the probability of the insurgence of corrosive phenomena. A surface breakdown delivers phenomenal amounts of energy onto the metal surface and therefore it was expected that the passivation layer on the metal was to suffer greatly when struck with such intensity. Results shown below confirmed this hypothesis. The separate contribution to of the three channels (RGB) to the final composed image, as described in the Experimental Procedures, are reported. Firstly, the reference sample, passivated but not exposed to a surface breakdown is shown in Fig. 4.

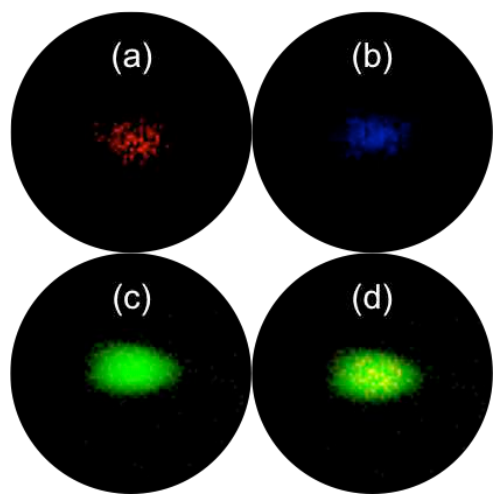

Fig. 4. SSIMS imaging of the section of a copper microwire $(\varnothing 25 \mu \mathrm{m})$ embedded in glass and passivated with Irgamet ${ }^{\circledR} 39$ in oil showing the intensity of copper (a), sulphur (b) and passivator (c) respectively and the resulting composed image $(\mathrm{d})$.

Looking at the intensity of each of the single image feeds separately it was possible to observe how the intensity of (c) 
appeared to be the greatest. This was consistent with a passivated copper surface, uniformly covered by tolyltriazole molecules. This can be appreciated in the composed picture (d), where the contribution from the active moiety of Irgamet ${ }^{\circledR} 39$ is even more obvious. For comparison, the results shown in Fig. 5 are very different.

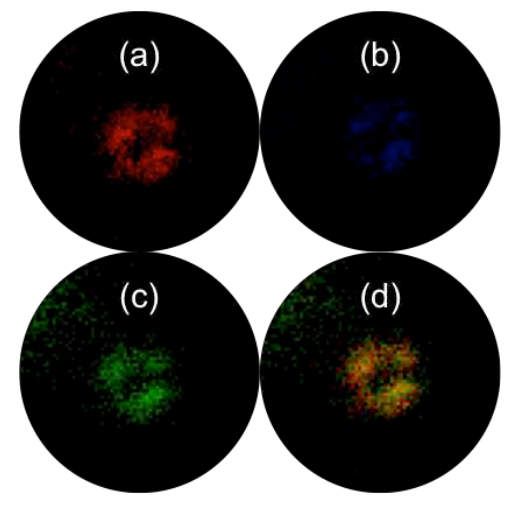

Fig. 5. SSIMS imaging of the section of a copper microwire (Ø $25 \mu \mathrm{m})$ embedded in glass, passivated with $\operatorname{Irgamet}^{\mathbb{}} 39$ and subject to a surface breakdown in oil showing the intensity of copper (a), sulphur (b) and passivator (c) respectively and the resulting composed image (d).

As expected, as a consequence of the amount of energy released on the surface of copper during the surface breakdown, the intensity of the passivator detected in (c) decreased decisively. On the contrary, more copper was detected (a) while sulphur remained substantially unchanged. The composed image (d) once again completes the results. These results clearly showed how the passivation layer could be negatively influenced by electric phenomena inside a transformer. Localized destruction of the passivation layer could cause the development of pit corrosion that could then expand, especially towards the hottest parts of the windings, as discussed in Fig. 3.

\section{CONCLUSIONS}

Static secondary ion mass spectrometry was demonstrated to be an analytical tool highly applicable to power transformer problems. Its intrinsic high molecular selectivity and the possibility to reprocess data into easily interpretable images opened a wide range of applications. SSIMS data clearly addressed the issues related to the lack of direct observation of the molecular passivation layer formed by $\operatorname{Irgamet}^{\circledR} 39$ on copper in insulating transformer oil. The observation, on the metal surface, of the tolyltriazole molecular fingerprint, confirmed experimentally the hypothesized mechanism of action of this passivator. SSIMS imaging of copper samples from a scrapped autotransformer ultimately proved its applicability to real-life situations as well as highlighted the close relationship between temperature gradient and passivator distribution on the windings of a transformer in service. Finally, the negative effect of a surface breakdown in oil on the passivation layer was investigated, revealing that these phenomena may indeed jeopardize the integrity of Irgamet ${ }^{\circledR 3} 9$ passivation layer. The exploitation of this unconventional advanced chemical technique could help understanding the influence of operating conditions and design of a transformer on the passivation layer at the molecular level and could lead to significant improvements of both operative guidelines and rating criteria for power transformers.

\section{Acknowledgments}

The authors gratefully thank National Grid for the financial support to the project. MF thanks BASF for providing Irgamet ${ }^{\circledR} 39$ and Dr H. Ding (Doble Engineering Company) for the collection of the transformer samples.

\section{References}

[1] F. Scatiggio et al., "Corrosive Sulfur Induced Failures in Oil-Filled Electrical Power Transformers and Shunt Reactors," IEEE Trans. Power Deliv., vol. 24, no. 3, pp. 1240-1248, 2009.

[2] Lukic, J. et al., "CIGRE WG A2-40 Copper sulphide long-term mitigation and risk assessment," 2013.

[3] P. Wiklund, M. Levin, and B. Pahlavanpour, "Copper Dissolution and Metal Passivators in Insulating Oil," IEEE Electr. Insul. Mag., vol. 23, no. 4, pp. 6-14, Jul. 2007.

[4] M. Dahlund et al., "CIGRE WG A2-32 Copper sulphide in transformer insulation Final Report," 2009.

[5] T. Amimoto et al., "Identification of affecting factors of copper sulfide deposition on insulating paper oil," IEEE Trans. Dielectr. Electr. Insul., vol. 16, no. 1, pp. 265-272, 2009.

[6] V. Tumiatti, C. Roggero, M. Tumiatti, S. Di Carlo, and R. Maina, "State of the art in quantification of DBDS and other corrosive sulfur compounds in unused and used insulating oils," IEEE Trans. Dielectr. Electr. Insul., vol. 19, no. 5, pp. 1633-1641, 2012.

[7] J. M. Lukic, S. B. Milosavljevic, and A. M. Orlovic, "Degradation of the Insulating System of Power Transformers by Copper Sulfide Deposition: Influence of Oil Oxidation and Presence of Metal Passivator," Ind. Eng. Chem. Res., vol. 49, no. 20, pp. 9600-9608, 2010

[8] H. Kawarai et al., "Role of dissolved copper and oxygen on copper sulfide generation in insulant oil," IEEE Trans. Dielectr. Electr. Insul., vol. 16, no. 5, 2009.

[9] R. M. De Carlo, M. C. Bruzzoniti, C. Sarzanini, R. Maina, and V. Tumiatti, "Copper contaminated insulating mineral oils-testing and investigations," IEEE Trans. Dielectr. Electr. Insul., vol. 20, no. 2, pp. 557-563, Apr. 2013.

[10] P. S. Amaro et al., "X-Ray Fluorescence as a Condition Monitoring Tool for Copper and Corrosive Sulphur Species in Insulating Oil," IEEE Trans. Dielectr. Electr. Insul., 2014, In Press.

[11] F. Scatiggio, M. Pompili, and R. Bartnikas, "Oils with presence of corrosive sulfur: mitigation and collateral effects," in IEEE Electrical Insulation Conference, 2009, pp. 478-481.

[12] R. Maina, V. Tumiatti, F. Scatiggio, M. Pompili, and R. Bartnikas, "Transformers Surveillance Following Corrosive Sulfur Remedial Procedures," IEEE Trans. Power Deliv., vol. 26, no. 4, pp. 23912397, 2011.

[13] M. C. Bruzzoniti, R. M. De Carlo, C. Sarzanini, R. Maina, and V. Tumiatti, "Determination of copper in liquid and solid insulation for large electrical equipment by ICP-OES. Application to copper contamination assessment in power transformers.," Talanta, vol. 99, pp. 703-11, Sep. 2012.

[14] M. Facciotti et al., "Contact-based corrosion mechanism leading to copper sulphide deposition on insulating paper used in oil-immersed electrical power equipment," Corros. Sci., vol. 84, pp. 172-179, Jul. 2014.

[15] R. Maina, V. Tumiatti, M. Pompili, and R. Bartnikas, "Corrosive sulfur effects in transformer oils and remedial procedures," IEEE Trans. Dielectr. Electr. Insul., vol. 16, no. 6, pp. 1655-1663, 2009.

[16] C. Bengtsson et al., "Oil corrosion and conducting Cu2S deposition in power transformer windings," CIGRE, Paris, 2006. 